\title{
Human thought controlled electrical switching using fast Fourier transform
}

\author{
Prof. Ganesh S. Deshmukh ${ }^{1}$, Bhushan P. Rathod ${ }^{2}$, Tejas S. Zagade ${ }^{3}$, Nitin D. Parkhe ${ }^{4}$, Paresh L. Waghmare ${ }^{5}$ \\ Pimpri Chinchwad College of Engineering, Nigdi, Pune India ${ }^{1}$ \\ Students, Pimpri Chinchwad College of Engineering, Nigdi, Pune, India ${ }^{2,3,4,5}$
}

\begin{abstract}
A brain-computer interface (BCI) is a new communication medium between the human brain and a digital world. BCI is emerging technology which can be used at every corner. The ambitious goal of a BCI technology can be the restoration of movements, communication, and environmental control for physically challenged people. An Electroencephalogram (EEG) based brain-computer interface will be connected with a system in order to control home application. It offers an alternative to natural communication and control. It is an artificial system that bypasses the body normal communication pathways. Different brain states are the result of different patterns of neural interaction. These patterns lead to waves characterized by different amplitudes and frequencies. Every interaction between neurons creates a minuscule electrical discharge. This project deals with the signals from the brain, as the different brain states are the result of different patterns of neural interaction. The signal generated by brain will be received by the brain wave sensor and it will divide into packets and the packet data transmitted to the wireless medium. The wave measuring unit will receive the brain wave raw data and it will convert into digital signal using MATLAB GUI platform. The interrupt generated will be sent to the microcontroller to operate the modules. The system operates with condition of appliance is based on changing with user's cognitive state.
\end{abstract}

Keywords: BCI (Brain Computer Interface), EEG (Electroencephalograph), FFT (Fast Fourier Transform).

\section{INTRODUCTION}

Brain-Computer Interface (BCI) device turns your which will used for physical challenging people, which are brainwaves into actions, unlocking new worlds of not move from one place to another. We can use a noninteractivity. BCI gives the details of user's mental state in invasive BCI device such as headset, which is use for the Attention and Meditation level, with the raw signal and information about the brainwave frequencies. The device can be used to interact with home applications. From the past, people are getting more interested in brain and the different activities happening in the brain, Neuroscience research has greatly helped us in our knowledge about the brain activities, and the electrical signals emitted by neurons in the brain. The patterns and frequencies of these electrical signals can be measured by placing a electrodes on different portions of the head. The Brain Computer Interface (BCI) Device measures the analog electrical signals, emitted as brainwaves, and processes them into digital signals. By measuring these different signals we can use them for any different purpose like controlling home appliances of electrical device such as lamps, fans, television etc.

This paper deals with the environmental control using BCI (Brain Computer Interface) technology. Brain computer interface provide severally movement disable people with alternative channel to communicate and to control simple devices. Human brain is the complex system of the world. BCI is a system that captures the brain activity in the form of EEG signal and translates to specific feature of the signal that represents the intent of the user into computer readable commands. This command can control and operate an electrical device, but in the human brain there is several things are going on at a time, that time brain wave classification needed.

In this paper our main aim to develop a human thought controlled electrical switching in our daily environment capturing the EEG wave from brain. Then we process those signal using MATLAB platform for generation of state such as Attention, eye-blink to remotely control multiple home appliances[1]-[3].

\section{LITERATURE SURVEY}

The author [5]has describe the details about brain computer interface and their different types like Invasive Brain Computer Interfaces, Partially Invasive Brain Computer Interfaces, Non Invasive Brain Computer Interfaces, Also their applications, recent developments and open problems in brain computer interface area of research.

EEG waves are classified with the help of Discrete Wavelet Transform (DWT) and with Fast Fourier Transform (FFT) by adopting the normalized EEG data [9]. For classification if EEG wave frequencies, DWT are used, while for implementing the EEG wave in multiresolution of DWT, Fast Fourier transform is used.

In our previous study [7]-[8], we found some feature extraction and classification technique for EEG signal. EEG signals are recorded from 16 channels and studied during mental task of the person. Feature extraction is carried out from these signals using several methods like Time analysis, Frequency Analysis, TimeFrequency Analysis, and Time-Frequency-Space Analysis. And these extracted signals are classified. interrupt for Electrical Switching using user's cognitive 
In paper [10], author has described the representation details about the EEG signal using MATLAB. With the help of MATLAB graphical user interface (GUI), EEG dataset and brain signal can be processed flexibly. Different brain signals are represented by comparing, analysing and simulating databases which is already loaded in the MATLAB software to process the EEG signals.

In paper [1], author has used non invasive brain computer interface (BCI) for controlling home devices. The Electroencephalographic signal (EEG) recorded from the brain activity using Emotive EPOCH headset are interfaced with the help of mouse emulator to a graphical user interface (GUI) on the computer screen. The user will use this GUI to control various devices in a home.

\section{PROPOSED MODEL}

BCI is an Emerging technology \& has wide range of uses in all the fields. This technology can be helpful in improving the quality of human life beings in general and elderly and disabled people. The device will capture the different brain wave signals from user's brain and will translate them into digital signal so that user can read those signals on the computer, then according to the different parameter set, the action will be performed. To implement a Brain Computer Interface for home, to remotely control multiple home appliances. The ambitious goal of a BCI is finally the restoration of movements, communication, and environmental control for handicapped people.

The proposed system will give the ability to user to control the home appliances with the help of brain computer interface system. The appliances can be controlled with the eye blink [13]-[14], concentration level, and meditation level. The proposed system will able to display the various EEG wave forms along with the user's eye blink status, user's concentration level.

\section{SYSTEM ARCHITECTURE}

Figure 1 shows an overview of the main components in the system design. The system flow is described below, followed by a component specification.

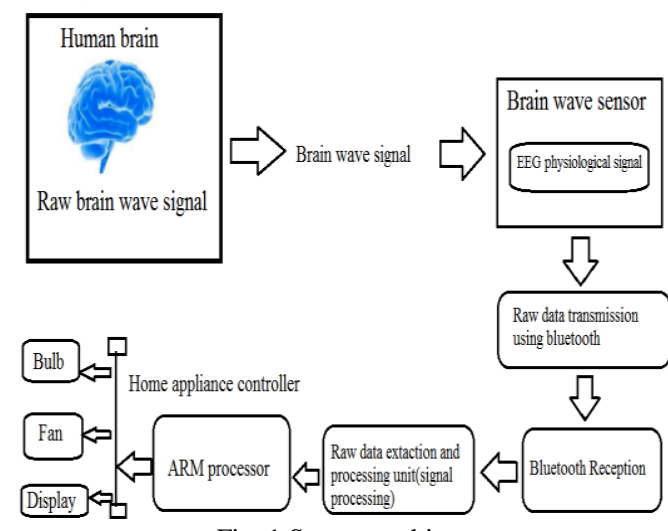

Fig. 1.System architecture
Bluetooth module to the personal computer using brain wave sensor headset. This measures EEG signal from human brain. Another Bluetooth module is used as shown in Fig. This module receives commands from the wireless device and directs them to the microcontroller. ARM LPC2148 microprocessor as shown in Fig 1. is used to process commands sent by the subjects.

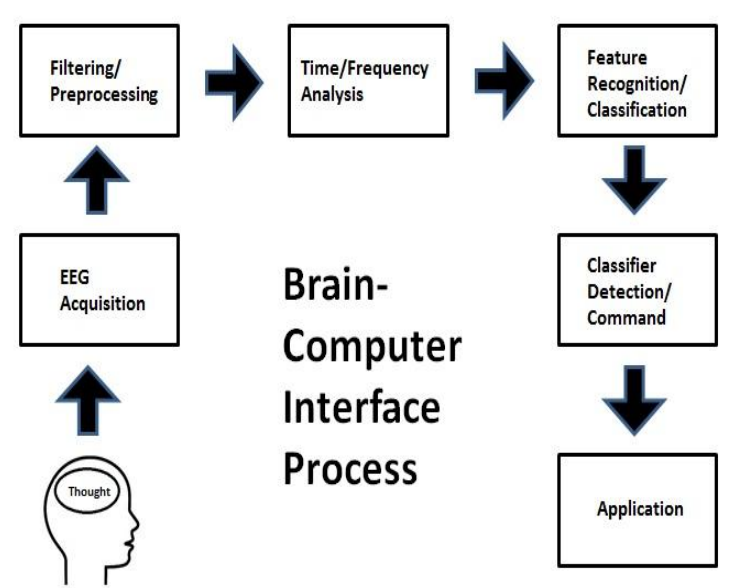

Fig. 2.Block diagram of BCI

Comparing these components with the BCI process flow in figure, we see that Brain wave sensor, Bluetooth module, corresponds to "brain signal input"; MATLAB interface corresponds to "EEG signal processing" and EEG signal classification" and ARM processor to "Electrical switching".

A BCI is a communication system between the user's brain and a certain application performed in a computer, and like any communication system has an input (e.g. EEG activity from the user's brain) an output (e.g. device commands), components that translate Input into output (translation device) and a protocol that control the timing of the operations. Figure 2 shows a basic dissection of a BCI system, and the interaction between its parts. The input of the BCI system is achieved by the certain bio-recording technology, described in Figure 2 as the Signal Acquisition Module. This input is processed to extract distinctive features (Feature Extraction Module), which should be able to separate the existing classes after being classified by Translation Algorithm module, which outputs a device command to control the Output Device or Application module. These parts of standard BCI systems, plus an off-line analysis (feature selection) are described in the following subsections.

\section{Different phases in Brain computer Interfacing:}

- $\quad$ Signal Acquisition

- $\quad$ Signal Pre-processing

- $\quad$ Signal Classifications

- Computer Interaction

As shown in Fig. 1, EEG raw signal from the user scalp is The unit will have the ability to collect raw brain wave collected, amplified, digitized and transmitted through a signal transmitted by brain wave sensor. 
a) The control/processing unit will be able to receive raw brain wave signals and will be able to extract and interpret instructions to be sent to the microcontroller.

b) The microcontroller within the control unit will issue its command to the electrical appliances through a simple control circuit.

c) The control unit will control the electrical appliances.

\section{A. EEG Signal Acquisition}

The operations that are performed by Brain Wave sensors are capturing of Brains electrical activities i.e. Electroencephalogram (EEG).The Raw Brain Wave signals are capture by Brain wave sensors from Human brain waves. The BCI headset is mounted on the scalp and the electrode which is a sensor is placed at forehead. This Raw EEG data is transmitted to PC or Laptop by using Bluetooth

\section{B. Signal Processing}

Signal processing consists of two essential parts. Feature extraction and translation. The former is preprocessing of signal by transforming it into a set of features; the latter translates them into desired commands for computer.

Feature extraction

In this part, we will discuss various approaches to feature extraction[8]-[11], which is basically an effort to reduce dimensions of measured data (or data stream when processing on-line which is exactly the case of BCI) so that comparing and classifying is simplified enough to be done real-time. Another important role of feature extraction is reducing the noise, so that most of information in the signal can be controlled by user.

In practical uses, the BCI signal we process has a form of discrete time series. There is an algorithm called "Discrete Fourier Transform (DFT), which transforms a particular discrete time series into a function of frequencies. The computation of DFT for a sample has the computational complexity of $\mathrm{O}\left(\mathrm{N}^{2}\right)$ (where $\mathrm{N}$ is a number of data points in a sample) which is not a efficient computation. There is a "Fast Fourier Transform (FFT)" that does much same as DFT, but it has a computational complexity of $\mathrm{O}\left(\mathrm{N} \log _{2} \mathrm{~N}\right)$.

In order to analyze the data signal for its frequency content, it has to be converted from the time domain into the frequency domain. This can be accomplished by applying a mathematical method known as Fourier Transform. In the context of EEG analysis, the Fourier Transform is applied (once for each channel) to a finite number of discrete data points belonging to successive sometimes overlapping) segments of equal length.

\section{Signal classification}

The FFT has been used for feature extraction and a threshold based rule for classification. Fast Fourier Transform is used in order to classify the signals according to their frequency Ranges [10].
EEG Signals can be classified as shown in table 1 below:

TABLE 1

$\begin{array}{ll}\text { Band } & \text { Frequency }(\mathrm{Hz}) \\ \text { Delta } & 0.5-3 \\ \text { Theta } & 3-8 \\ \text { Alpha } & 8-12 \\ \text { Beta } & 12-30 \\ \text { Gamma } & 30+\end{array}$

Delta: It tends to be the highest in amplitude and the slowest waves. It is seen normally in adults in slow wave sleep.

Theta: Theta is seen normally in young children. It may be seen in drowsiness or arousal in older children and adults; it can also be seen in meditation.

Alpha: It emerges with closing of the eyes and with relaxation, and attenuates with eye opening or mental exertion.

Beta: It is seen usually on both sides in symmetrical distribution and is most evident frontally. Beta activity is closely linked to motor behavior.

Gamma: Gamma waves are thought to represent binding of different populations of neurons together into a network for the purpose of carrying out a certain cognitive or motor function.

We can use FFT to extract attention signal from raw EEG. Attention signal is the average intensity of the EEG signals in frequency 9 to $14 \mathrm{~Hz}$. More stable the extracted frequencies, the higher the intensity of attention signal. Average intensity of EEG signals is passed through filter and normalized them as attention grades from 0 to 100

\section{Controlling the devices}

Once the signals are classified, signals can be used for the controlling certain appliances based on threshold value set for the interrupt generation. Interrupt will be provided to the microcontroller. According to the type of interrupt received, microcontroller will operate the particular device such as fan, bulb etc.

\section{Conclusion}

The main goal of this paper is to design and implement a human thought controlled electrical switching system using BCI technology for the physically challenged people. Non invasive BCI method is used for capturing the brain signals with the help of brain wave sensor. Different cognitive state of the user like attention level, meditation level, eye-blink can be used to control different home appliance in real time. Feature extraction and classification is carried out of EEG signals, and those are classified with the help of Fast Fourier Transform (FFT). The signals are classified according their respective frequencies ranging from $0.5 \mathrm{~Hz}$ to $30 \mathrm{~Hz}$. By setting the threshold value for an event, the interrupt is generated. This Interrupt is sent to the microcontroller unit for operating the appliances. 


\section{REFERENCES}

[1] Wei Tuck Lee,Humaira Nisar,Aamir S. Malik,Kim Ho Yeap, “A Brain Computer Interface for Smart Home Control",IEEE 17th International Symposium on Consumer Electronics (ISCE), 2013.

[2] Chin-Teng Lin, Fellow, Bor-Shyh Lin, Fu-Chang Lin and Che-Jui Chang"Brain Computer Interface-Based Smart Living Environmental Auto-Adjustment Control System in UPnP Home Networking” IEEE Systems Journal, Vol. 8, No. 2, June 2014.

[3] Chin-Teng Lin, Fu-Chang Lin, Shi-An Chen, Shao-Wei Lu, Te-Chi Chen, Li-Wei Ko, "EEG-based Brain-computer Interface for Smart Living Environmental Auto-adjustment", Journal of Medical and Biological Engineering, 30(4): 237-245, Jun 2010

[4] Wolpaw, J.R.,"Brain-computer interface research comes of age: traditional assumptions meet emerging realities." Journal of motor behavior, November 2010.

[5] Anupama.H.S,N.K.Cauvery,Lingaraju.G.M, "Brain computer interface and its types - a study", International Journal of Advances in Engineering \& Technology, ISSN: 2231-1963, May 2012.

[6] T. Kameswara Rao, M. Rajyalakshmi, Dr. T. V. Prasad, "An Exploration on Brain Computer Interface and Its Recent Trends", International Journal of Advanced Research in Artificial Intelligence, Vol. 1, No. 8, 2012.

[7] Vasudha Vashisht, Dr. T. V. Prasad, Dr. S.V.A.V. Prasad, "Technology Boon: EEG Based Brain Computer Interface - A Survey", International Journal of Computer Science and Mobile Computing, Vol. 2, Issue. 4, April 2013.

[8] M. Rajya Lakshmi, Dr. T. V. Prasad Dr. V. Chandra Prakash,"Survey on EEG Signal Processing Methods" ,International Journal of Advanced Research in Computer Science and Software Engineering, Volume 4, Issue 1, January 2014.

[9] Maan M. Shaker, "EEG Waves Classifier using Wavelet Transform and Fourier Transform" International Journal of Medical, Health, Pharmaceutical and Biomedical Engineering Vol:1 No:3, 2007.

[10] Sasikumar Gurumurthy, Vudi Sai Mahit, Rittwika Ghosh, "Analysis and simulation of brain signal data by EEG signal processing technique using MATLAB", International Journal of Engineering and Technology (IJET), ISSN: 0975-4024 Vol 5 No 3 Jun-Jul 2013.

[11] M.Balamareeswaran, P.Meenakshi Vidya, K.S.Sujatha "Optimal Signal Processing and Transmission for Brain Computer Interface" Proceeding of 5th National Conference on VLSI, Embedded, and Communication \& Networks on April 17, 2014.

[12] Kenji Nakayama Yasuaki Kaneda Akihiro Hirano, "A Brain Computer Interface Based on FFT and Multilayer Neural Network Feature Extraction and Generalization", International Symposium on Intelligent Signal Processing and Communication Systems, 2007.

[13] Jzau Sheng Lin, Win Ching Yang,"Wireless Brain-computer Interface for Electric Wheelchairs with EEG and eye-blinking signals "International Journal of Innovative Computing, Information and Control Volume 8, Number 9, September 2012.

[14] K. S. Ahmed"Wheelchair Movement Control VIA Human Eye Blinks"American Journal of Biomedical Engineering p-ISSN: 2163-1050 e-ISSN: 2163-1077, 2011. 